\title{
Is the effect of prior exercise on postprandial lipaemia the same for a moderate-fat meal as it is for a high-fat meal?
}

\author{
Nicholas M. Hurren, Frank F. Eves and Andrew K. Blannin* \\ School of Sport and Exercise Sciences, University of Birmingham, Edgbaston, Birmingham B15 2TT, UK
}

(Received 22 April 2010 - Revised 29 July 2010-Accepted 1 September 2010-First published online 15 November 2010)

\begin{abstract}
Moderate-intensity exercise can lower the TAG response to a high-fat meal; however, the British diet is moderate in fat, and no study to date has compared the effect of such exercise on responses to high-fat and moderate-fat meals. The present work investigated the effect of brisk walking performed $13 \mathrm{~h}$ before intake of both high-fat and moderate-fat meals on postprandial plasma TAG concentrations. Eight inactive, overweight men completed four separate $2 \mathrm{~d}$ trials, i.e. rest (Con) or a 90-min treadmill walk (Ex) on the evening of day 1 , followed by the ingestion of a moderate-fat (Mod) or high-fat (High) meal on the morning of day 2. High-fat meals contained $66 \%$ of total energy as fat, while the percentage was $35 \%$ for moderate-fat meals; both the meals were, however, isoenergetic. On day 2 , venous blood was sampled in the fasted state, 30 and $60 \mathrm{~min}$ after ingesting the test meal and then hourly until $6 \mathrm{~h}$ post-meal. Exercise reduced plasma TAG concentrations significantly $(P<0 \cdot 001)$, with no exercise $\times$ meal interaction $(P=0 \cdot 459)$. Walking reduced the total TAG response to a high-fat meal by $29 \%$ (relative to High Con); the same bout of exercise performed before ingesting a moderate-fat meal lowered total TAG by $26 \%$ (compared with Mod Con). The ability of a single moderate-intensity aerobic exercise bout to lower postprandial TAG concentrations is just as great, in percentage terms, when the test meal ingested is of a moderate rather than a high fat content.
\end{abstract}

Key words: Acute exercise: Postprandial lipaemia: Plasma TAG: Carbohydrate

In healthy men, free from CVD, plasma TAG concentrations late in the postprandial period have been shown to correlate positively with intima-media thickness of the common carotid artery, a marker of early atherosclerosis ${ }^{(1)}$. When coupled with epidemiological data showing that individuals with elevated non-fasting TAG concentrations are at greater risk of cardiac events ${ }^{(2,3)}$, there is evidence to suggest that an exaggerated TAG response to a fat meal is a risk factor for CHD. Performing aerobic exercise before ingesting a fat-containing meal has been shown on many occasions to lower the extent of the subsequent lipaemia $^{(4)}$; however, the fat content of the test meal used may not be relevant in the context of meals consumed as part of the habitual diet.

WHO guidelines recommend that fat should contribute $15-30 \%$ of an individual's daily energy intake, although it is acknowledged that highly active individuals consuming diets rich in fruit and vegetables will not necessarily risk unhealthy weight gain (and associated chronic disease risks) when fat represents $35 \%$ of energy intake ${ }^{(5)}$. A government-commissioned survey of British men, aged 19-64 years, found that dietary fat consistently accounted for $35-36 \%$ of energy intake across age groups ${ }^{(6)}$; however, almost all studies investigating the effects of exercise on postprandial lipaemia have used test meals that contain more than $60 \%$ of energy as fat ${ }^{(4)}$. Therefore, it could be argued that the use of test meals containing far higher fat percentages than seen on average in the British diet has artificially magnified any differences between exercise and control trials in such studies. A small number of studies have investigated how postprandial lipaemia is affected when exercise is performed before ingesting a meal containing $35-45 \%$ of energy as $\mathrm{fat}^{(7-15)}$; unfortunately, these studies do not agree in their conclusions, and the differing protocols used make it difficult to explain why the results are not more uniform. Due to the discrepant nature of findings from studies using moderate-fat meals, it is possible that the beneficial effects of physical activity in reducing postprandial TAG concentrations have been overstated. While a cross-sectional study could compare the effect of exercise on the response to a moderate-fat meal in one group and a high-fat meal in another group, the question of whether exercise is as potent in lowering lipaemia when the fat content of the meal is reduced

Abbreviations: HF walk, walk before the ingestion of a high-fat meal; MF walk, walk before the ingestion of a moderate-fat meal. 
cannot truly be answered unless the responses to both meals are considered in the same individuals. No such work has been published. The present study therefore investigated the effect of 90 min of prior treadmill walking on postprandial lipaemia after ingesting meals of both high and moderate fat content in the same participants. Elevated postprandial lipaemia has been shown to be more common among certain population sub-groups, with maleness ${ }^{(16,17)}$, ageing $^{(18)}$, inactivity $^{(19,20)}$ and obesity ${ }^{(21-23)}$ being particular risks; therefore it is particularly relevant to recruit people with several of these risk factors as a population in which to investigate potential interventions. As a result, middle-aged, inactive, overweight men were recruited, due to their increased risk of developing CVD.

\section{Methods \\ Participants}

Eight non-smoking, inactive, overweight men (age $47 \cdot 0$ (SD 9.1) years, height $179(\mathrm{SD} 7) \mathrm{cm}$, body mass 92.7 (SD 4.8$) \mathrm{kg}$, BMI $29 \cdot 2(\mathrm{SD} 2 \cdot 3) \mathrm{kg} / \mathrm{m}^{2}$, body fat 32.9 (SD $6 \cdot 1) \%$ and estimated maximal oxygen uptake $\left(\mathrm{VO}_{2 \max }\right)$ $37 \cdot 5$ ( $\mathrm{SD} 5 \cdot 3$ ) $\mathrm{ml} / \mathrm{kg}$ per min) participated in the study. Inactivity was defined as participation in $1 \mathrm{~h}$ or less of structured physical activity per week, and overweight were classed as a BMI in excess of $25 \mathrm{~kg} / \mathrm{m}^{2}$. None of the men was known to have CVD or diabetes, and each potential participant's general practitioner was contacted to ensure that the study did not present a particular danger to the patient's health based on his medical history. Participation in the study was not permitted until written general practitioner approval was received. All men known to be taking medication affecting lipoprotein metabolism were excluded from participating in the study. The present study was conducted according to the guidelines laid down in the Declaration of Helsinki, and all procedures involving human participants were approved by the School of Sport and Exercise Sciences Local Ethics SubCommittee, University of Birmingham. Written informed consent was obtained from all the participants.

\section{Study design}

Investigative trials followed a $2 \mathrm{~d}$ approach with an exercise session or rest during the evening of day 1 and an oral fat tolerance test on the morning of day 2 . To compare the effect of walking $v$. control on the response to both the high-fat and moderate-fat meals, the participants completed four trials: a high-fat exercise trial, a high-fat control trial, a moderate-fat exercise trial and a moderate-fat control trial. Oral fat tolerance tests were separated by a minimum of $4 \mathrm{~d}$. Trial order was counterbalanced to control for the effect of serial position in which the trial occurred, with each participant following a different test order. The participants were asked to keep a diary of all food and drink consumed on the day before, and the day of, their first trial; this diet was then replicated before all subsequent trials. The participants agreed not to perform any physical activity beyond that required for activities of daily living during the $2 \mathrm{~d}$ leading up to an oral fat tolerance test, and ingestion of alcohol and caffeine was prohibited for $24 \mathrm{~h}$ before each walk/evening rest.

\section{Preliminary exercise testing}

Before the main trials, all the participants performed a submaximal incremental treadmill test. The men were given 5 min to become familiarised with walking on the treadmill and were asked to select a speed which they felt confident of maintaining for $90 \mathrm{~min}$, but which would represent a moderate effort for them. Once a speed was agreed between participant and experimenters (with heart rate generally in the range of $100-110$ beats $/ \mathrm{min}$ ), the first 5-min walking stage began with the treadmill at $0 \%$ gradient. Heart rate (Polar Vantage NV; Polar Electro Oy, Kempele, Finland) and expired gas measurements were made during the fifth minute of each 5-min stage, and the participants were asked to supply a rating of perceived exertion (RPE) from a Borg RPE Scale ${ }^{(24)}$ at the conclusion of each stage. The treadmill gradient was increased by $2-3 \%$ at the end of each 5-min stage, with the test being terminated upon participants reaching $85 \%$ of their individual predicted maximal heart rate. The equation used to predict maximal heart rate was $208-0.7 \times$ age $^{(25)}$. Heart rate was subsequently plotted against oxygen uptake, and a prediction of individual maximal oxygen uptake $\left(\mathrm{VO}_{2 \max }\right)$ was made via extrapolation of a linear plot (trendline). Oxygen uptake $v$. treadmill gradient plots were then made, and the equation of the slope was used to determine the gradient required to elicit $60 \%$ of $\mathrm{VO}_{2 \max }$ for each man.

\section{Main trials}

Aerobic exercise trials. The participants arrived at the laboratory at 18.00 hours. They then walked for $90 \mathrm{~min}$ at the speed selected in the preliminary test and at a treadmill gradient calculated to evoke $60 \%$ of individual predicted $\mathrm{VO}_{2 \max }$. To confirm the $\mathrm{VO}_{2}$ at which the men were actually working, expired breath samples were collected during the last 2 min of each sixth of the walk, i.e. spaced at $15-\mathrm{min}$ intervals. On occasion, subtle alterations to the treadmill gradient were made to ensure that oxygen uptake remained as close to $60 \%$ of $\mathrm{VO}_{2 \max }$ as possible. Heart rate was monitored continuously throughout the trial, with values stored electronically, at 5-s intervals, for subsequent download.

Control trials. During control trials, all the participants remained seated or supine for a period equivalent in length to the exercise trials. The men were allowed to perform paperwork, watch television/DVDs, work on a computer or read a book, but no physical activity above that required to perform these actions was permitted. 


\section{Body fat estimation}

All the participants had skinfold measurements taken during their final trial to estimate body fat percentage. Measurements were made at the bicep, tricep, subscapular and suprailiac sites by an International Society for the Advancement of Kinanthropometry-accredited kinanthropometrist; log values were then entered into age-specific four-site skinfold equations ${ }^{(26)}$ to calculate body density. Body density values were converted to body fat percentages using the equation of Siri ${ }^{(27)}$.

\section{Evening (post-intervention) meal}

After exercising or resting in the laboratory (i.e. day 1), all the participants were provided with an evening meal of red pepper and mozzarella stacks, spaghetti bolognese and strawberry smoothie yoghurts. This meal contained 36\% of energy as fat, $46 \%$ as carbohydrate and $18 \%$ as protein, with the percentage of energy as fat guided by governmental guidelines for a balanced diet ${ }^{(28)}$. The total energy content of the meal provided for the men was calculated based on each individual's habitual evening energy intake. During the week before their first trial, the participants were asked to keep a detailed food diary for three consecutive evenings; total energy counts for each evening were made using the nutrition information available on the packaging for the foods consumed and the nutrition software package 'Comp-eat' (Comp-eat version 5.0.1). A mean of the energy counts for the three evenings was taken, and this value represented the total quantity of energy provided to the participant. Evening meals were consumed within $1 \mathrm{~h}$ of finishing a treadmill walk/rest period, after which the men were asked to fast until the following morning. The mean energy provided by the meal was 4.41 (SD 0.50) MJ (1055 (SD 120) kcal).

\section{Oral fat tolerance tests}

The participants arrived at the laboratory at 08.00 hours after an overnight fast (i.e. day 2). A cannula was inserted into an antecubital or forearm vein, and a fasting blood sample $(5 \mathrm{ml})$ was drawn. The participants were then taken to the research kitchen where they were presented with the test meal. The high-fat meal provided $1.2 \mathrm{~g}$ fat/ $\mathrm{kg}$ body mass, $1.2 \mathrm{~g}$ carbohydrate/ $\mathrm{kg}$ body mass and $0.2 \mathrm{~g}$ protein/kg body mass (66\% fat, 29\% carbohydrate and $5 \%$ protein as percentage of total energy) and consisted of a liquid component containing whole milk, double cream and sugar, along with a solid component of hazelnut cereal bars. The moderate-fat meal provided $0.64 \mathrm{~g}$ fat $/ \mathrm{kg}$ body mass, $1.93 \mathrm{~g}$ carbohydrate/kg body mass and $0.74 \mathrm{~g}$ protein/kg body mass (35\% fat, $47 \%$ carbohydrate and $18 \%$ protein as percentage of total energy) and comprised a drink containing whole milk, double cream, sugar and skimmed milk powder, along with apricot cereal bars.
The meals were isoenergetic (both providing $68.6 \mathrm{~kJ}$ of energy per kg body mass) and were designed such that the mass of cereal bar given in the different meals would be the same. The meals provided 6.36 (sEm 0.12) MJ (1520 (SEM 28) kcal) of energy, with fat loads of 111 (SEM 2) and 59 (SEM 1) g for the high-fat and moderate-fat meals, respectively. The percentage of fat accounted for by saturates was $54.7 \%$ for the high-fat meal and $54.8 \%$ for the moderate-fat meal. As a greater volume of liquid was present in the moderate-fat meal (due to it being less energy-dense than the high-fat liquid), a small volume of water (calculated on an individual basis) was provided for the participants along with the high-fat meal to ensure that the total volume of liquid consumed in each test meal was equal.

Venous blood samples were drawn 0.5, 1, 2, 3, 4, 5 and $6 \mathrm{~h}$ after the ingestion of the test meal, with the first $2 \mathrm{ml}$ of blood drawn at each time point being discarded as waste. The cannula was kept patent by flushing every 20 min with non-heparinised saline. The participants were given no further food and were permitted to drink only water during the postprandial period. Water was provided ad libitum during the first trial, with the volume being recorded and replicated in the remaining three trials.

\section{Analytical procedures}

Blood samples were dispensed into precooled $\mathrm{K}_{3}$-EDTA vacutainers for recovery of plasma and into plain vacutainers for obtainment of serum. Blood contained within $\mathrm{K}_{3}$-EDTA tubes was centrifuged immediately, for $15 \mathrm{~min}$, at $4{ }^{\circ} \mathrm{C}$ and $3500 \mathrm{rpm}$. Plasma was then aspirated and divided into aliquots for immediate storage at $-80^{\circ} \mathrm{C}$. Uncoated vacutainers were left on the bench top for $30 \mathrm{~min}$ to allow clotting to occur before centrifugation. Plasma was analysed by enzymatic colourimetric methods using a ninety-six-well plate reader (Multiskan MS; Labsystems, Helsinki, Finland) for TAG (Sigma-Aldrich, Poole, UK) and using a centrifugal analyser (Cobas Mira Plus, Roche, Switzerland) for NEFA (Wako, Neuss, Germany) and glucose (ABX Diagnostics, Montpellier, France). Serum was analysed for insulin by ELISA (IDS Limited, Frankfurt am Main, Germany). Owing to the nature of the TAG assay, plasma glycerol concentrations were also measured to allow for correction of TAG concentrations. The samples were stored at $-80^{\circ} \mathrm{C}$ until immediately before analysis, with all samples for each participant being analysed in the same run. Within-batch coefficients of variation were $2.2 \%$ for TAG (after correction for plasma glycerol), $1.0 \%$ for NEFA, $1.8 \%$ for glucose and $5 \cdot 2 \%$ for insulin.

\section{Calculations and statistics}

The total energy expended during 90-min treadmill walks was calculated using indirect calorimetry, with the 
assumption that $1 \mathrm{~g}$ of fat and carbohydrate yield $40 \cdot 8 \mathrm{~kJ}$ $(9.75 \mathrm{kcal})$ and $17 \mathrm{~kJ}(4.07 \mathrm{kcal})$ of energy, respectively, when exercising at a moderate intensity ${ }^{(29)}$, and assuming that no protein was oxidised during the exercise. Substrate oxidation rates during exercise were calculated using equations for moderate- to high-intensity exercise (50-75\% of $\left.\mathrm{VO}_{2 \max }\right)^{(29)}$. Metabolite datasets were assessed for a normal distribution using the Shapiro-Wilk test; the datasets did not differ significantly from a normal distribution. Repeated-measures ANOVA was used to determine the effects of exercise (walk $v$. control), meal (high fat $v$. moderate fat) and time (0, 0.5, 1, 2, 3, 4, 5 and $6 \mathrm{~h}$ ) for all postprandial measures, as well as any interactions between the main effects. The first two orthogonal polynomial contrasts (linear and quadratic components) were computed simultaneously to further explain the differences between conditions across time. These analyses were performed using SYSTAT 11 for Windows. The majority of previous studies have used repeated-measures ANOVA followed by post hoc Tukey tests to assess the location of differences when the omnibus $F$ test has been significant. However, this approach has substantial limitations for repeatedmeasures designs. First, post hoc Tukey tests require independence of the means that they compare, something that is never the case in a repeated-measures design. Furthermore, as opposed to the appropriate $t$ tests with Bonferroni adjustment, Tukey's honestly significant difference test does not control the $\alpha$ rate satisfactorily under conditions where sphericity is violated ${ }^{(30,31)}$. As violation of sphericity is the rule rather than the exception in repeated-measures designs ${ }^{(32)}$, Stevens ${ }^{(33)}$ asserts that when $\boldsymbol{\epsilon}$ (sphericity) is less than 0.70 (where 1.00 reflects a spherical dataset; see also Maxwell ${ }^{(30)}$ ), Bonferroni adjustment, not the Tukey honestly significant difference test, should be used to conduct pairwise comparisons. Due to the multiple comparisons required to assess the differences between conditions across several time points, Bonferroni adjustment is not informative given the conservative nature of the procedure; in this situation, orthogonal polynomial contrasts provide an elegant solution. Orthogonal polynomials provide single degree of freedom comparisons of the waveform over time and hence do not violate the assumption of independence between means required for the use of post hoc Tukey tests. Linear components reflect the overall increases or decreases over time, whereas the quadratic components reflect any non-linear peak or trough that might occur within the time window. Logarithmic transformation of time was found to improve symmetry (a requirement of trend analysis via the use of orthogonal polynomial contrasts) for NEFA, glucose and insulin, but not for TAG; therefore, only the NEFA, glucose and insulin analyses were performed with time logged to the base $e$. To allow comparisons with previous work, areas under the curve representing the total and incremental lipaemic responses are also reported. The total area under the curve score was calculated as the $6 \mathrm{~h}$ area under the plasma TAG concentration $v$. time curve, using the trapezoidal rule. The incremental area under the curve score was calculated as the area under the curve normalised to the $0 \mathrm{~h}$ value; thus it is a true postprandial measure reflecting only the change in concentration after a meal. Two-way ANOVA (exercise $\times$ meal) was conducted on the total area under the curve scores, incremental area under the curve scores, fasting values and peak values. Variables measured during exercise were compared using paired samples $t$ tests. SPSS 16 for Mac was used for all such analyses. Relationships between the variables were examined using Pearson's product-moment correlation coefficient. Fasting concentrations of glucose and insulin were used to derive a validated surrogate measure of insulin resistance ${ }^{(34)}$. Specifically, fasting glucose and insulin concentrations were entered into a 'HOMA2 Calculator' downloaded from the Diabetes Trials Unit, University of Oxford web site $^{(35)}$. Statistical significance was accepted at the level of $P<0.05$. All the data are presented as means with their standard errors unless otherwise stated.

\section{Results}

\section{Responses during brisk walking}

During both the walk before a high-fat meal (HF walk), and the walk before a moderate-fat meal (MF walk), the participants walked at a speed of $5.7(\operatorname{sem} 0.2) \mathrm{km} / \mathrm{h}$ up a gradient of 4.2 (sem 0.5$) \%$. Mean oxygen uptake was $21.7(\operatorname{sem} 1 \cdot 1) \mathrm{ml} / \mathrm{kg}$ per $\min \left(58 \cdot 1(\operatorname{sem} 1 \cdot 1) \% \mathrm{VO}_{2 \max }\right)$ during the HF walk and 22.0 (SEM 1.0$) \mathrm{ml} / \mathrm{kg}$ per min (58.8 (sem 0.7$) \% \mathrm{VO}_{2 \max }$ ) during the MF walk. Gross energy expenditure was 3.75 (SEM 0.19 ) and 3.80 (sem 0.18) MJ during the HF and MF walks, respectively. Mean heart rate was 127 (SEM 5) beats/min during the HF walk and 128 (SEM 3) beats/min during the MF walk. Ratings of perceived exertion from the Borg RPE Scale were 14.3 (SEM 0.4) (HF) and 13.7 (SEM 0.7) (MF) for the walks; the exercise was therefore perceived to lie between the descriptors 'somewhat hard' and 'hard'. No significant differences were found between the two walks for any of the responses recorded.

\section{Plasma and serum concentrations in the fasted state}

Fasting plasma concentrations of TAG, NEFA and glucose and serum concentrations of insulin are presented in Table 1. Plasma TAG concentrations were reduced by $28 \cdot 1$ and $22 \cdot 1 \%$, compared with their respective controls, when walking was performed before the ingestion of a high-fat meal and a moderate-fat meal (effect of exercise, $P=0.001)$. Plasma NEFA concentrations, serum insulin concentrations and homeostasis model assessment scores were not found to be significantly different between the exercise and control conditions before the ingestion of either meal; 
Table 1. Plasma and serum concentrations in the fasted state for both walking and control trials before the ingestion of either a high-fat meal or a moderate-fat meal

(Mean values with their standard errors; $n 8$ )

\begin{tabular}{|c|c|c|c|c|c|c|c|c|}
\hline & \multicolumn{2}{|c|}{ Con High } & \multicolumn{2}{|c|}{ Con Mod } & \multicolumn{2}{|c|}{ Walk High } & \multicolumn{2}{|c|}{ Walk Mod } \\
\hline & Mean & SEM & Mean & SEM & Mean & SEM & Mean & SEM \\
\hline TAG (mmol/l) & 1.21 & 0.23 & 1.14 & 0.20 & $0.87^{\star \star \star}$ & 0.23 & $0.89^{\star \star \star}$ & 0.15 \\
\hline NEFA (mmol/l) & 0.33 & 0.03 & 0.32 & 0.03 & 0.37 & 0.03 & 0.33 & 0.02 \\
\hline Glucose (mmol/l) & $5 \cdot 84$ & 0.32 & 5.90 & 0.32 & $5 \cdot 38^{\star \star *}$ & 0.25 & $5 \cdot 69^{* \star *}$ & 0.28 \\
\hline Insulin (pmol//) & $82 \cdot 6$ & $17 \cdot 9$ & $100 \cdot 7^{*}$ & $19 \cdot 4$ & 83.4 & $19 \cdot 3$ & $103 \cdot 4^{*}$ & $20 \cdot 3$ \\
\hline HOMA & 1.56 & 0.35 & $1.94^{\star}$ & 0.38 & 1.59 & 0.36 & $1.94^{\star}$ & 0.38 \\
\hline
\end{tabular}

Con High, high-fat control trial; Con Mod, moderate-fat control trial; Walk High, walk before a high-fat meal; Walk Mod, walk before a moderate-fat meal; HOMA, homeostasis model assessment.

* Mean values were significantly different from the high-fat meal trials (main effect of meal to be ingested; $P<0.05$ ).

${ }^{* * *}$ Mean values were significantly different from the control trials (main effect of exercise; $P \leq 0.001$ ).

however, an effect of meal about to be ingested was found for insulin $(P=0.048)$ and homeostasis model assessment $(P=0 \cdot 048)$. Plasma glucose concentrations were significantly lower following exercise $(P=0 \cdot 001)$.

\section{Postprandial plasma and serum concentrations}

Plasma TAG concentrations (Fig. 1) were lower after walking trials than after control trials $(P<0 \cdot 001)$. No significant effect of meal was detected $(P=0 \cdot 295)$, and there was no interaction between the exercise and meal conditions $(P=0.459)$. A main effect of time $(P<0.001)$ was evident, with TAG concentrations increasing during the postprandial period for all the trials, and significant exercise $\times$ time $(P<0.001)$ and meal $\times$ time $(P=0.037)$ interactions were found. For the exercise $\times$ time interaction, $92 \%$ of the total variance was explained by a linear function $(P=0.001)$, with the slope of the increase in TAG over time being steeper for the control trials than for the walking trials. As the difference in TAG concentration between the exercise and control trials at the end of the postprandial period was greater than the difference before the ingestion

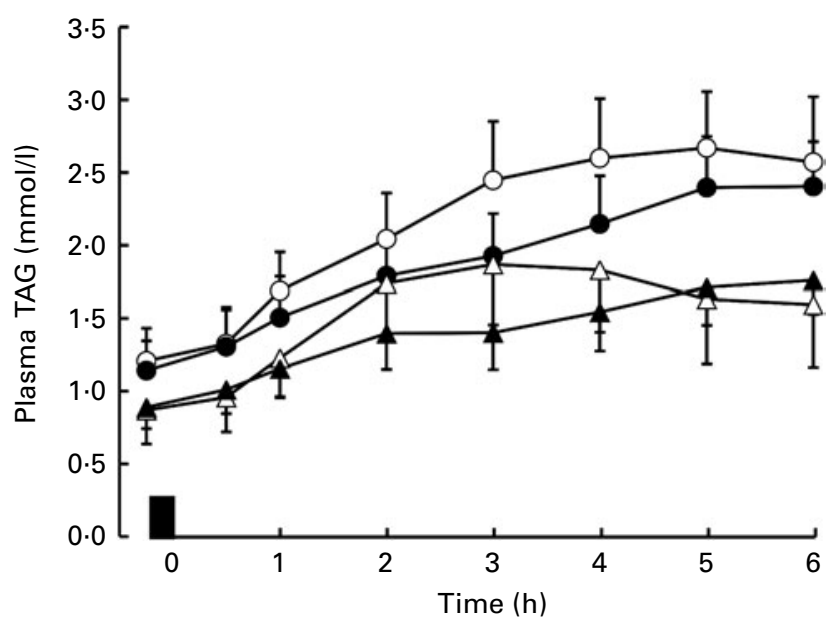

Fig. 1. Mean ( \pm SEM) plasma TAG concentrations in the fasted state and for $6 \mathrm{~h}$ after the ingestion of a high-fat meal $(O, \triangle)$ or a moderate-fat meal $(\bullet, \Delta)$ with either prior exercise $(\triangle, \boldsymbol{\Delta})$ or prior rest $(O, \bullet)$. $\mathbf{\square}$, Ingestion of the test meals. of the meals, it can be interpreted that TAG increased significantly more quickly in the control trials. For the meal $\times$ time interaction, $81 \%$ of the total variance was explained by a quadratic function $(P=0 \cdot 009)$. Fig. 2 shows the change in plasma TAG concentration from baseline across time, therefore allowing for clearer discernment of differences between the trials due to meal intake. The significant meal $\times$ time interaction, largely explained by a quadratic function, suggests that TAG concentrations peaked earlier after ingesting a high-fat meal than after ingesting a moderate-fat meal. Specifically, mean TAG concentration can be observed to peak at $3 \mathrm{~h}$ in the high-fat walking trial and at $5 \mathrm{~h}$ in the high-fat control trial, before falling, whereas TAG concentrations continued to rise until $6 \mathrm{~h}$ in the moderate-fat meal trials. Peak TAG values were substantially reduced with exercise $30.0 \%$ lower for high-fat meal and $26.9 \%$ lower for moderate-fat meal; $P=0.001$ for effect of exercise), but meal fat concentration did not have an impact on the peak values observed (effect of meal; $P=0 \cdot 245$ ).

Total and incremental lipaemic responses are shown in Fig. 3. Total lipaemic responses to the high-fat and

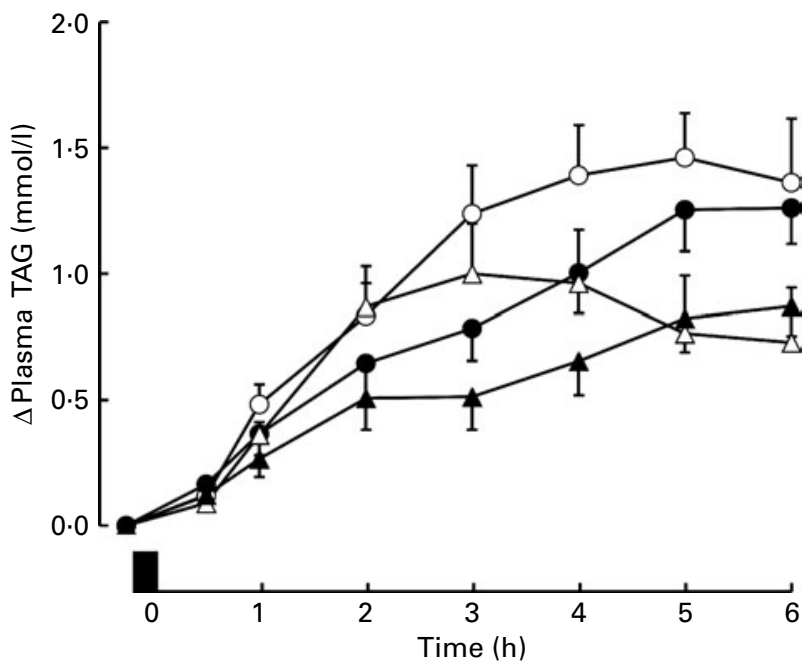

Fig. 2. Changes in mean ( \pm SEM) plasma TAG concentrations in the $6 \mathrm{~h}$ after the ingestion of a high-fat meal $(O, \triangle)$ or a moderate-fat meal $(\bullet, \mathbf{\Delta})$ with either prior exercise $(\triangle, \boldsymbol{\Delta})$ or prior rest $(\bigcirc, \boldsymbol{\bullet})$. $\mathbf{\square}$, Ingestion of the test meals. 


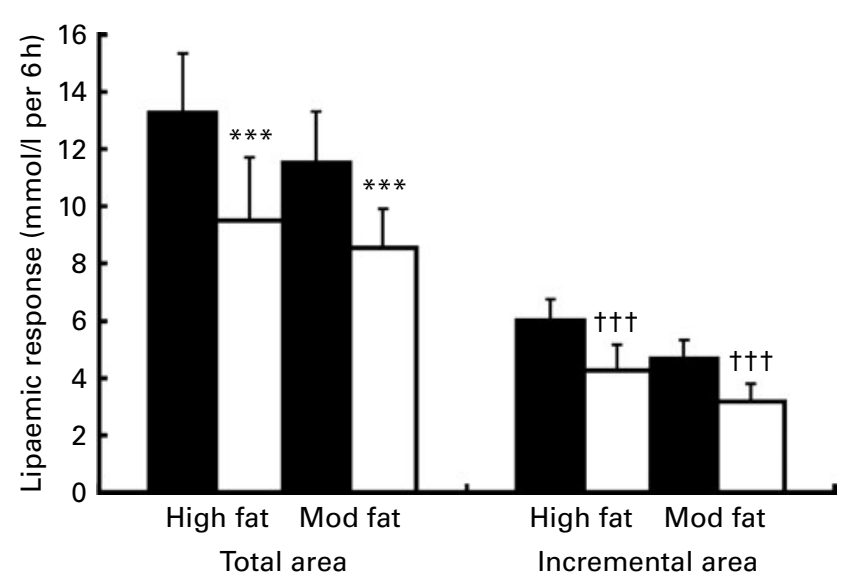

Fig. 3. Area under the curve (AUC) scores showing the total and incremental lipaemic responses. High-fat meal responses are shown by the two bars on the left for each area, with moderate-fat meal responses represented by the bars on the right. Control trials are shown as $\mathbf{\square}$, whereas walking trials are shown as $\square$. ${ }^{\star \star *}$ Main effect of exercise for total AUC, $P<0.001$. ††† Main effect of exercise for incremental AUC, $P \leq 0.001$.

moderate-fat meals were reduced with exercise by 28.5 and $26.0 \%$, respectively $(P<0 \cdot 001)$. Incremental lipaemic responses to both the meals were also suppressed with prior walking (suppression of $29 \cdot 1 \%$ for high-fat meal and $31.7 \%$ for moderate-fat meal; $P=0.001$ for effect of exercise). Neither the total nor the incremental lipaemic responses were lower after the ingestion of moderate-fat meals than after the ingestion of high-fat meals $(P=0.226$ and 0.075 , respectively, for effect of meal). Fasting TAG concentrations in each of the four trials were positively correlated with TAG total area under the curve $(r$ 0.9570.990 , all $P<0.001)$, TAG incremental area under the curve $(r$ 0.745-0.924; all $P<0.05)$ and peak TAG values ( $r$ 0.930-0.974, all $P \leq 0.001$ ).

Plasma NEFA concentrations (Fig. 4) tended to be higher after exercise, but not to a significant extent $(P=0 \cdot 078)$. NEFA concentrations changed considerably with time

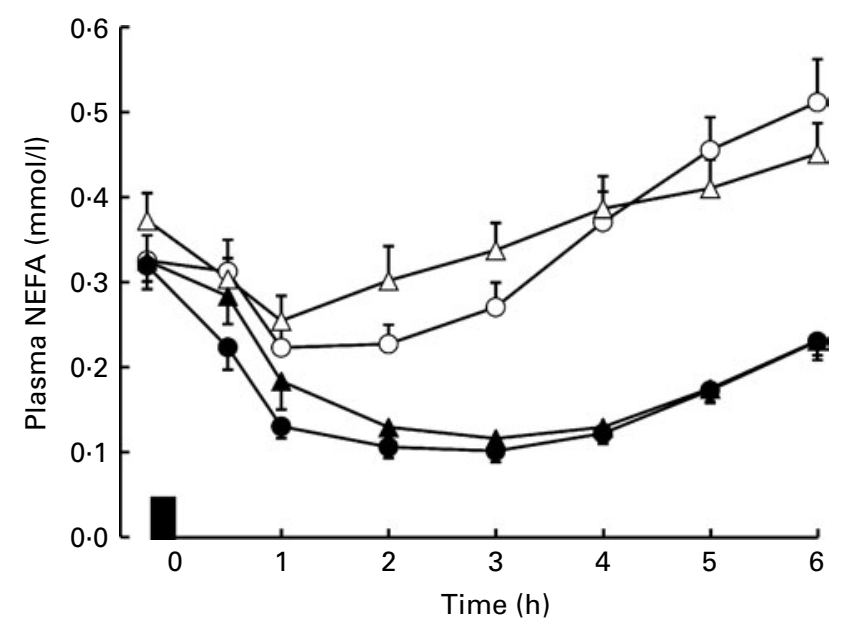

Fig. 4. Mean ( \pm SEM) plasma NEFA concentrations in the fasted state and for $6 \mathrm{~h}$ after the ingestion of a high-fat meal $(O, \triangle)$ or a moderate-fat meal $(\bullet, \boldsymbol{\Delta})$ with either prior exercise $(\triangle, \boldsymbol{\Delta})$ or prior rest $(\bigcirc, \boldsymbol{\bullet})$. $\mathbf{\square}$, Ingestion of the test meals.
$(P<0 \cdot 001)$ and were substantially higher after the ingestion of high-fat meals than after the ingestion of moderate-fat meals $(P<0 \cdot 001)$. Additional to this, the shape of the NEFA curves was different between the meals (meal $\times$ time interaction; $P<0 \cdot 001$ ). All the conditions showed an initial reduction in concentration compared with the fasting values. However, NEFA concentrations reached a nadir after $1 \mathrm{~h}$ in the high-fat meal trials before rebounding to above baseline by $4 \mathrm{~h}$, whereas the concentrations continued to decline until $3 \mathrm{~h}$ following the ingestion of moderate-fat meals and did not return to baseline within $6 \mathrm{~h}$. The slope of the overall change in concentration was positive for the high-fat meal trials and negative for the moderate-fat meal trials such that $97 \%$ of the variation of the meal $\times$ time interaction was attributable to a linear component $(P=0 \cdot 001)$.

Plasma glucose concentrations (Fig. 5) did change significantly during the postprandial period (effect of time; $P=0 \cdot 015$ ), but there were no significant main effects of exercise or meal, nor were there any significant interactions between main effects $(P>0.05$ for all)

Postprandial serum insulin concentrations (Fig. 6) were lower with the walking trials than with the control trials $(P=0.019)$ and were lower with high-fat meals than with moderate-fat meals $(P=0.032)$. In the early postprandial period, insulin concentrations rose sharply for all four conditions, but decreased steadily towards baseline by $6 \mathrm{~h}$ (effect of time; $P=0.003$ ); a quadratic function accounted for $78 \%$ of the variation in insulin concentration across time $(P=0.004)$. A significant exercise $\times$ time interaction was present $(P=0.023)$, with a quadratic function explaining $32 \%$ of the variation $(P=0 \cdot 023)$. Related to this, insulin concentrations were observed to peak earlier in the walking trials than in the control trials $(1 v .2 \mathrm{~h})$. Following the ingestion of high-fat meals, the insulin concentration curves showed much shallower arcs, with lower peak values than after the ingestion of moderate-fat meals.

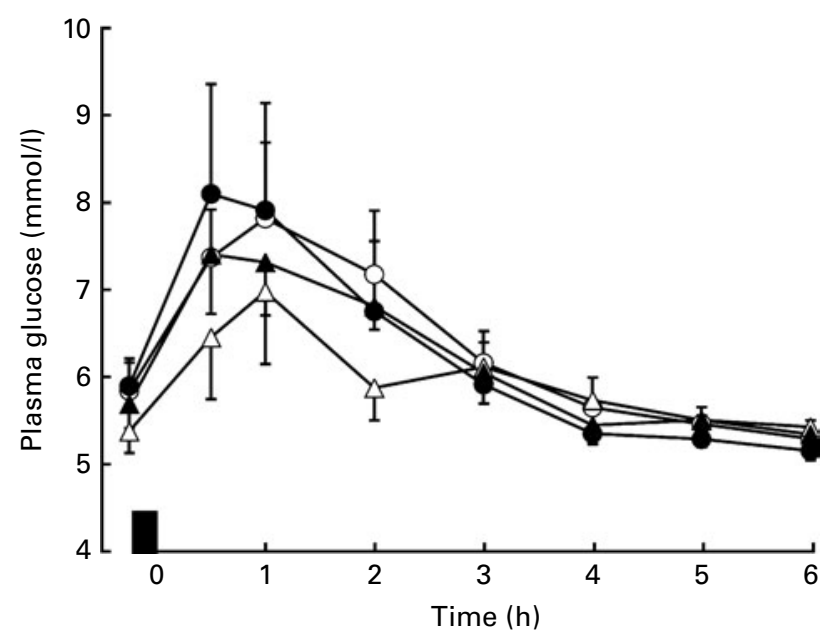

Fig. 5. Mean ( \pm SEM) plasma glucose concentrations in the fasted state and for $6 \mathrm{~h}$ after the ingestion of a high-fat meal $(\bigcirc, \triangle)$ or a moderate-fat meal $(\bullet, \mathbf{\Delta})$ with either prior exercise $(\triangle, \boldsymbol{\Delta})$ or prior rest $(O, \bullet)$. $\mathbf{\square}$, Ingestion of the test meals. 


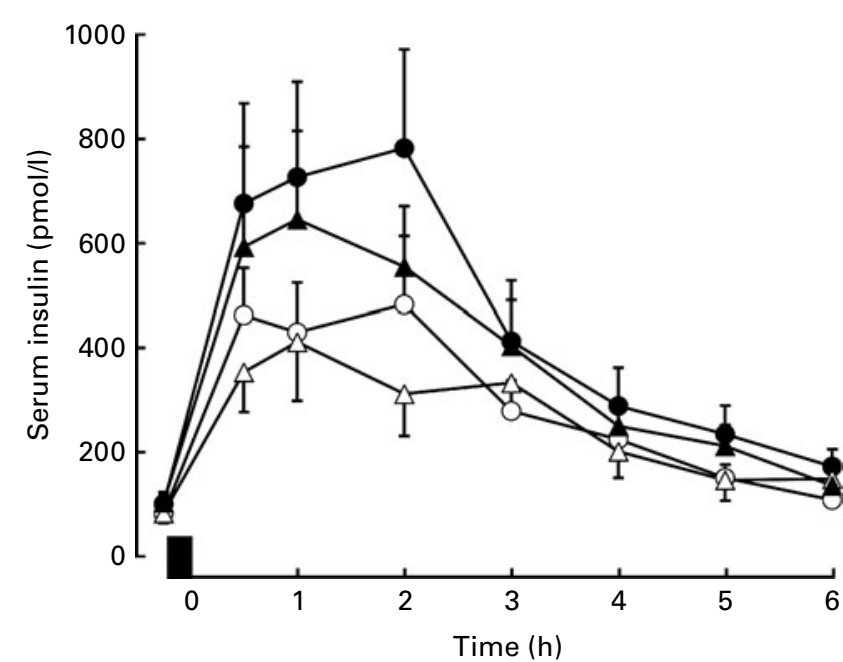

Fig. 6. Mean ( \pm SEM) serum insulin concentrations in the fasted state and for $6 \mathrm{~h}$ after the ingestion of a high-fat meal $(O, \triangle)$ or a moderate-fat meal $(\bullet, \boldsymbol{\Delta})$ with either prior exercise $(\triangle, \boldsymbol{\Delta})$ or prior rest $(\bigcirc, \boldsymbol{\bullet})$. $\boldsymbol{\square}$, Ingestion of the test meals.

This was reflected by a significant meal $\times$ time interaction within the quadratic component of the curves $(P=0.029)$.

\section{Discussion}

The main finding of the present study was that a brisk 90-min treadmill walk reduced the postprandial lipaemia observed after the ingestion of meals of both high and moderate fat content to very similar extents in percentage terms, relative to a control trial for each meal. Secondly, we report that isoenergetic meals of high and moderate fat content exert distinct effects upon the plasma TAG concentration observed at different stages during the postprandial period, and that the differential time courses of postprandial lipaemia after ingesting these meals can be observed to diverge further when the added stimulus of prior exercise is present. Thirdly, within a group of eight middle-aged, overweight, inactive men, the total and incremental lipaemic responses to a moderate-fat meal are not significantly different from those to a high-fat meal, regardless of whether prior exercise is performed or not.

The present study was designed to conclusively answer the question of whether prior moderate-intensity exercise lowers the postprandial lipaemia brought on by a moderate-fat meal as effectively as the postprandial lipaemia observed after the ingestion of a high-fat meal. Our data support the idea that a single brisk walk, performed on the evening before ingesting a meal containing either a moderate or a high percentage of energy as fat, will attenuate fasting and postprandial TAG concentrations. The substantial and significant reduction in incremental lipaemic response after walking (with no exercise $\times$ meal interaction) provides evidence that prior exercise exerts a true postprandial effect in limiting the increase in plasma TAG concentrations observed after the ingestion of both the moderate-fat and high-fat meals.
All the participants in the present study were overweight or obese as defined by BMI, with body fat percentages supporting the idea that extra mass was predominantly fat. Several studies using overweight/obese individuals have shown a decrease in postprandial lipaemia when prior exercise was performed $10-18 \mathrm{~h}$ before ingesting either a high-fat meal ${ }^{(22,36-41)}$ or a moderate-fat meal ${ }^{(13,15)}$. Three other studies have reported no attenuation of postprandial lipaemia when overweight/obese individuals exercised $15-18 \mathrm{~h}$ before ingesting a high-fat meal ${ }^{(42-44)}$; however, the fact that the participants were also type 2 diabetic in two of the three studies ${ }^{(42,43)}$ may partly account for such findings. The statistically significant reduction in postprandial lipaemia observed in the present study, in which the participants walked $13 \mathrm{~h}$ before meal consumption, is therefore in agreement with the majority of the published literature.

Postprandial TAG concentrations increased over time during the walking trials in the present study, but the increase was not as large as in the control trials. The smaller increase after exercise could reflect an increased clearance rate of TAG from the plasma, a decrease in secretion of VLDL-TAG from the liver or even a reduced rate of appearance of chylomicron-TAG into the circulation. Based on our data, it is not possible to elucidate which mechanism was most important, although the $13 \mathrm{~h}$ delay between the end of the treadmill walk and the ingestion of the test meals would perhaps make it less likely for an attenuated rate of appearance of chylomicron-TAG to be a substantial factor. Indirect evidence suggests that the lowering of postprandial TAG concentrations after moderate exercise is most likely to result from a reduced secretion of VLDL-TAG into the plasma ${ }^{(45)}$, although stable isotope studies conducted in the fasting state do not appear to offer support for this mechanism ${ }^{(46,47)}$.

While postprandial lipaemia was not lower with moderate-fat meals than with high-fat meals, there were differences in the shape of the TAG curves with the two meals. Essentially, the high-fat response showed an increase, peak and decline within the $6 \mathrm{~h}$ period of blood sampling, whereas the moderate-fat response displayed a near linear increase. As studies collecting samples for more than $6 \mathrm{~h}$, after exercise and control trials, have not shown peak TAG concentrations occurring after $6 \mathrm{~h}$ for a high-fat meal ${ }^{(22,37-39,43,48-50)}$, it appears sensible to conclude that TAG concentrations would continue to fall at time points beyond $6 \mathrm{~h}$ of high-fat meal intake in the present study. For the moderate-fat meal trials, the rate of TAG increase appears to be reduced towards the end of the $6 \mathrm{~h}$ postprandial period; however, as peak values were recorded at $6 \mathrm{~h}$, it cannot be said with certainty that TAG concentrations would not have continued to increase if further samples had been collected. That said, studies where blood samples were collected for $8 \mathrm{~h}$ after the ingestion of a single moderate-fat meal have shown peak TAG values at $5-6 h^{(7,9)}$. The TAG concentration in the late 
postprandial period may be of pathophysiological importance due to its positive correlation with a surrogate measure of early atherosclerosis ${ }^{(1)}$. Therefore, the observation made within the present study of an elevated TAG concentration late in the postprandial period after ingesting a moderate-fat meal could have clinical relevance. Addition of carbohydrate to a fat load has previously been shown to slow gastric emptying and to produce a later peak in plasma TAG concentration ${ }^{(51,52)}$; our data illustrate that when two isoenergetic meals with different carbohydrate, fat and protein contents are consumed, peak TAG concentration is delayed after ingesting the meal with the higher carbohydrate content.

While the moderate-fat meal contained only $53 \%$ of the fat-derived energy of the high-fat meal, it was found that without prior exercise, the rise in plasma TAG concentration (across the $6 \mathrm{~h}$ postprandial period, relative to baseline) after the ingestion of the moderate-fat meal was $77.5 \%$ of the rise observed with the high-fat meal. When prior exercise was performed, a near identical situation was observed, with the increase in TAG after the ingestion of the moderate-fat meal being $74.6 \%$ of the high-fat meal rise. Our finding that the incremental TAG response to the moderate-fat meals was proportionally greater than the response to the high-fat meals (relative to the dietary fat load given) is supported by data showing no difference in incremental TAG response to isoenergetic meals containing $44.5,65.2$ and $73.9 \%$ of energy as fat ${ }^{(53)}$. Similarly, when the incremental TAG area under the curve was calculated after ingesting virtually isoenergetic meals with $91 \cdot 2$ and $55.6 \%$ of energy as fat, TAG concentrations were significantly increased after the ingestion of both the meals, without a significant difference between the trials ${ }^{(54)}$. An earlier study, in type 2 diabetic patients, reported that the postprandial TAG response to a mixed meal containing $25 \%$ of energy as fat was only marginally, and nonsignificantly, less than that to an isoenergetic meal containing $45 \%$ fat $^{(55)}$. The protein content of the two meals given by Chen et al. was kept constant (15\% of energy), therefore implicating the higher carbohydrate content of the lower-fat meal as a factor preventing an attenuated lipaemic response. Others have found positive correlations between the quantity of fat ingested and either the resulting postprandial lipaemia ${ }^{(56-58)}$ or the peak TAG concentration ${ }^{(59)}$; however, the meals provided in these studies were not isoenergetic and therefore the impact of the greater energy intake with the higher-fat meals cannot be discounted as an influencing factor in the more pronounced lipaemic response. If the energy content of two meals is kept balanced, the available evidence suggests that postprandial lipaemia will not be significantly different between the two meals, even if the fat content of one is higher.

The lack of kinetic data in the present study precludes thorough investigation of the mechanisms responsible for the increase in postprandial TAG concentration out of proportion with the intake of dietary fat. However, potential mechanisms include increased esterification of NEFA to TAG by the liver after the ingestion of the moderate-fat meals due to the greater insulin response ${ }^{(60)}$; reduced clearance of TAG into skeletal muscle after the ingestion of the moderate-fat meals due to insulin downregulating skeletal muscle lipoprotein lipase activity ${ }^{(61,62)}$; or increased de novo lipogenesis after the ingestion of the moderate-fat meals, with the newly synthesised fatty acids becoming incorporated into VLDL-TAG ${ }^{(63)}$. Further studies using the combination of stable isotope tracers and biopsies, or arterio-venous difference measurements, are required before more conclusive answers can be reached regarding the specific mechanism behind this observation.

The greater NEFA response in the high-fat meal trials agrees with previous data regarding the response to isoenergetic meals containing different quantities of carbohydrate and fat ${ }^{(53)}$. Insulin is known to inhibit adipose tissue lipolysis ${ }^{(64)}$, and as the insulin response in our study was greater for moderate-fat meals than for high-fat meals, this offers a plausible mechanism for the lower NEFA response after the moderate-fat meals. Additionally, infusing insulin along with a high-fat mixed meal has been shown to increase re-esterification of lipoprotein lipase-derived fatty acids in adipose tissue compared with ingesting the meal alone ${ }^{(65)}$, therefore offering a second mechanism through which the moderate-fat meals could lower postprandial NEFA concentrations. Insulin can also slightly lower the activity of lipoprotein lipase within skeletal muscle ${ }^{(61)}$, therefore reducing hydrolysis of circulating TAG. Reduced breakdown of TAG passing through skeletal muscle capillaries could theoretically reduce spillover of exogenous fatty acids. This may not occur in practice, however, as trapping of fatty acids released when TAG is cleaved by lipoprotein lipase within skeletal muscle has been reported as close to $100 \%$ in the $6 \mathrm{~h}$ period after mixed meal ingestion ${ }^{(66)}$. The fat load of this meal was lower than either of the meals provided in the present study; therefore, it is not clear whether fatty acid spillover from skeletal muscle would be increased if a greater fat load were present. Due to the lack of kinetic data in our study, we are unable to estimate the contribution of these three mechanisms to the lowered plasma NEFA response after moderate-fat meal intake.

Fasting glucose concentrations were significantly reduced for the walking trials, but this did not translate into an improvement in insulin sensitivity as calculated using the updated homeostasis model assessment ${ }^{(34)}$. Fasting insulin concentrations and homeostasis model assessment scores were significantly higher for the trials where a moderate-fat meal was about to be ingested; however, as no test meal had been ingested at this point, the reason for this difference is unclear. There does not appear to be a cephalic insulin response brought on when anticipating food intake ${ }^{(67)}$ and the magnitude of 
the insulin response immediately after eating (before glucose has entered the blood) is similar for carbohydrateand fat-rich meals ${ }^{(68)}$, therefore we consider the pulsatility of insulin in the fasted state the most likely explanation. Insulin is released in short-term high-frequency pulses, occurring every 6-15 $\min ^{(69-72)}$. As only a single blood sample was taken in the fasted state for each participant during each trial, it is possible that the fasting serum insulin concentration could be at the top of a peak before a moderate-fat meal (following a recent pulse of insulin), but at the bottom of a trough before high-fat meal intake. Drawing three fasting blood samples (spaced 5 min apart) in which to measure insulin concentration may have prevented what we believe is a chance finding.

Postprandial glucose concentrations were not different between the walking and control trials, but as postprandial insulin concentrations were significantly lower for the walking trials (for both meals), it is quite possible that postprandial insulin sensitivity was favourably altered in the exercise trials. However, we found no relationship between any measures of insulin and TAG (all correlations, $P>0.05)$, and retrospective analysis of datasets from several studies found that the exercise-induced changes in TAG and insulin (in both the fasted and postprandial states) were not related ${ }^{(73)}$.

The energy content of the high-fat meal in our study was guided by prior publications in the field, with energy from each macronutrient provided per kg of body mass. However, as the participants in the present study were heavier than in most previous work, the energy provided by the meals was also greater. Indeed, the meals provided approximately $61 \%$ of the recommended daily energy intake for an average man. We acknowledge that the energy content of the meals in the present study was high and this should be considered when drawing comparisons with other work; however, it should also be noted that, regardless of the high energy counts, none of the men failed to finish the meals. While we believe the percentage of energy from fat used in the lower-fat meal in the present study (35\%) is justifiably moderate, the actual quantity of fat present in this meal was higher than that in previous studies using moderate-fat meals, due to the greater overall energy content. The current findings may not represent the response for women, owing to the fact that only men participated in the present study; therefore further research using female participants is necessary. As the majority of the previous literature has shown reductions in postprandial lipaemia after exercise using young individuals, it is likely that young men would respond in the same way as the middle-aged men in the present study; however, additional investigations are required before it can be said with certainty that our findings would be replicated in both young and elderly men. The available evidence suggests that lean and obese individuals derive the same lipaemic benefit (in percentage terms) from exercising before ingesting a high-fat meal ${ }^{(22)}$, but a comparative study would need to be performed before it could be said conclusively that lean individuals would show the same response to a moderate-fat meal as observed with the participants in the present study.

In conclusion, a 90-min brisk walk reduced the postprandial lipaemia associated with meals of both high and moderate fat content. The percentage reduction in postprandial lipaemia with walking was very similar for the two different meals when compared with control trials. TAG concentrations were not significantly lower after a moderate-fat meal was ingested instead of a high-fat meal. The time course of changes in plasma TAG was, however, different between the meals, with high-fat meals tending to prompt an earlier TAG peak. These findings suggest that when a meal contains a substantial quantity of other macronutrients (particularly carbohydrate), it does not require a substantial dietary fat load to elevate plasma TAG concentrations in a group of middle-aged, overweight, inactive men. Furthermore, exercising before ingesting a meal may be a more promising intervention to offset postprandial lipaemia than reducing the fat content of the diet.

\section{Acknowledgements}

We thank Dr Oliver Witard for taking skinfold measurements during the study and the participants for giving up their time to allow the research to take place. N. M. H. and A. K. B. co-designed the study, recruited the participants, collected the data and analysed the biochemical samples. N. M. H. performed the statistical analysis and drafted the manuscript. F. F. E. was involved in statistical analysis of the data, interpretation of such analysis and writing of the manuscript. A. K. B. was involved in editing and writing the manuscript and was responsible for overall supervision of the study. No external funding towards this research was received by any of the authors; all the costs were met by the academic research budget of the corresponding author (A. K. B.). The authors declare that they have no conflict of interest regarding any aspect of this research.

\section{References}

1. Karpe F, de Faire U, Mercuri M, et al. (1998) Magnitude of alimentary lipemia is related to intima-media thickness of the common carotid artery in middle-aged men. Atherosclerosis 141, 307-314.

2. Nordestgaard BG, Benn M, Schnohr P, et al. (2007) Nonfasting triglycerides and risk of myocardial infarction, ischemic heart disease, and death in men and women. JAMA 298, 299-308.

3. Bansal S, Buring JE, Rifai N, et al. (2007) Fasting compared with nonfasting triglycerides and risk of cardiovascular events in women. JAMA 298, 309-316.

4. Malkova D \& Gill JMR (2006) Effects of exercise on postprandial lipoprotein metabolism. Future Lipidol 1, 743-755. 
5. Joint WHO/FAO Expert Consultation on Diet, Nutrition and the Prevention of Chronic Diseases, (2002: Geneva, Switzerland) \& World Health Organization (2003) Diet, Nutrition and the Prevention of Chronic Diseases: Report of a Joint WHO/FAO Expert Consultation. Geneva: World Health Organization.

6. Henderson L, Gregory J, Irving K, et al. (2003) The National Diet and Nutrition Survey: Adults Aged 19 to 64 Years. vol. 2: Energy, Protein, Carbohydrate, Fat and Alcohol Intake. London: The Stationery Office.

7. Kolifa M, Petridou A \& Mougios V (2004) Effect of prior exercise on lipemia after a meal of moderate fat content. Eur J Clin Nutr 58, 1327-1335.

8. Petridou A, Gerkos N, Kolifa M, et al. (2004) Effect of exercise performed immediately before a meal of moderate fat content on postprandial lipaemia. BrJ Nutr 91, 683-687.

9. Kokalas N, Petridou A, Nikolaidis MG, et al. (2005) Effect of aerobic exercise on lipaemia and its fatty acid profile after a meal of moderate fat content in eumenorrhoeic women. $\mathrm{BrJ}$ Nutr 94, 698-704.

10. Pfeiffer M, Ludwig T, Wenk C, et al. (2005) The influence of walking performed immediately before meals with a moderate fat content on postprandial lipemia. Lipids Health Dis $\mathbf{4}, 24$.

11. Pfeiffer M, Wenk C \& Colombani PC (2006) The influence of 30 minutes of light to moderate intensity cycling on postprandial lipemia. Eur $J$ Cardiovasc Prev Rehabil 13, 363-368.

12. James AP, Slivkoff-Clark K \& Mamo JC (2007) Prior exercise does not affect chylomicron particle number following a mixed meal of moderate fat content. Lipids Health Dis $\mathbf{6}, 8$.

13. Burton FL, Malkova D, Caslake MJ, et al. (2008) Energy replacement attenuates the effects of prior moderate exercise on postprandial metabolism in overweight/obese men. Int J Obes (Lond) 32, 481-489.

14. Miyashita M \& Tokuyama K (2008) Moderate exercise reduces serum triacylglycerol concentrations but does not affect pre-heparin lipoprotein lipase concentrations after a moderate-fat meal in young men. Br J Nutr 99, 1076-1082.

15. Miyashita M (2008) Effects of continuous versus accumulated activity patterns on postprandial triacylglycerol concentrations in obese men. Int J Obes (Lond) 32, 1271-1278.

16. Heller FR, Vandenplas C, Desager JP, et al. (1993) The vitamin A fat-loading test in young normolipidemic subjects. Clin Chim Acta 219, 167-176.

17. Kolovou GD, Anagnostopoulou KK, Pavlidis AN, et al. (2006) Metabolic syndrome and gender differences in postprandial lipaemia. Eur J Cardiovasc Prev Rehabil 13, 661-664.

18. Jackson KG, Knapper-Francis JM, Morgan LM, et al. (2003) Exaggerated postprandial lipaemia and lower post-heparin lipoprotein lipase activity in middle-aged men. Clin Sci (Lond) 105, 457-466.

19. Hardman AE, Lawrence JE \& Herd SL (1998) Postprandial lipemia in endurance-trained people during a short interruption to training. J Appl Physiol 84, 1895-1901.

20. Dixon NC, Hurst TL, Talbot DCS, et al. (2009) Active middleaged men have lower fasting inflammatory markers but the postprandial inflammatory response is minimal and unaffected by physical activity status. J Appl Physiol 107, 63-68.

21. Lewis GF, O'Meara NM, Soltys PA, et al. (1990) Postprandial lipoprotein metabolism in normal and obese subjects: comparison after the vitamin A fat-loading test. J Clin Endocrinol Metab 71, 1041-1050.

22. Gill JM, Al-Mamari A, Ferrell WR, et al. (2004) Effects of prior moderate exercise on postprandial metabolism and vascular function in lean and centrally obese men. $\mathrm{J} \mathrm{Am} \mathrm{Coll} \mathrm{Cardiol}$ 44, 2375-2382.
23. Bartual A, González C, Martínez Hervás S, et al. (2006) Effect of gender and obesity on postprandial lipemia in nondiabetic normolipidemic subjects and subjects with familial combined hyperlipidemia. Rev Clin Esp 206, 213-219.

24. Borg GA (1982) Psychological bases of perceived exertion. Med Sci Sports Exerc 14, 377-381.

25. Tanaka H, Monashan KD \& Seals DR (2001) Age-predicted maximal heart rate revisited. J Am Coll Cardiol 37, 153-156.

26. Durnin JV \& Womersley J (1974) Body fat assessed from total body density and its estimation from skinfold thickness: measurements on 481 men and women aged from 16 to 72 years. Br J Nutr 32, 77-97.

27. Siri WE (1956) The gross composition of the body. Adv Biol Med Phys 4, 239-280.

28. Department of Health (1994) Nutritional Aspects of Cardiovascular Disease. Report of the Cardiovascular Review Group of the Committee on Medical Aspects of Food Policy. London: HMSO.

29. Jeukendrup AE \& Wallis GA (2005) Measurement of substrate oxidation during exercise by means of gas exchange measurements. Int J Sports Med 26, Suppl. 1, S28-S37.

30. Maxwell SE (1980) Pairwise multiple comparisons in repeated measures designs. J Educ Behav Stat 5, 269-287.

31. Baker SP \& Lew R (1987) A Monte Carlo comparison of Tukey's and Bonferroni's methods with an optimized Bonferroni multiple comparison procedure in repeated measures experiments. Proceedings of the Biopharmaceutical Section of the American Statistical Association, pp. 19-22.

32. Field AP (1998) A Bluffer's guide to sphericity. Newsletter of the Mathematical, Statistical and Computing Section of the British Psychological Society 6, 13-24.

33. Stevens J (1986) Applied Multivariate Statistics for the Social Sciences. Hillsdale, NJ: Lawrence Erlbaum Associates, Inc.

34. Levy JC, Matthews DR \& Hermans MP (1998) Correct homeostasis model assessment (HOMA) evaluation uses the computer program. Diabetes Care 21, 2191-2192.

35. HOMA2 Calculator, version 2.2. (C) Diabetes Trials Unit, University of Oxford. http://www.dtu.ox.ac.uk/index. php?maindoc $=/$ homa/ (last retrieved 24 March 2010).

36. Gill JM, Mees GP, Frayn KN, et al. (2001) Moderate exercise, postprandial lipaemia and triacylglycerol clearance. Eur J Clin Invest 31, 201-207.

37. Zhang JQ, Ji LL, Nunez G, et al. (2004) Effect of exercise timing on postprandial lipemia in hypertriglyceridemic men. Can J Appl Physiol 29, 590-603.

38. Zhang JQ, Ji LL, Fretwell VS, et al. (2006) Effect of exercise on postprandial lipemia in men with hypertriglyceridemia. Eur J Appl Physiol 98, 575-582.

39. Zhang JQ, Ji LL, Fogt DL, et al. (2007) Effect of exercise duration on postprandial hypertriglyceridemia in men with metabolic syndrome. J Appl Physiol 103, 1339-1345.

40. Mestek ML, Plaisance EP, Ratcliff LA, et al. (2008) Aerobic exercise and postprandial lipemia in men with the metabolic syndrome. Med Sci Sports Exerc 40, 2105-2111.

41. Harrison M, O'Gorman DJ, McCaffrey N, et al. (2009) Influence of acute exercise with and without carbohydrate replacement on postprandial lipid metabolism. J Appl Physiol 106, 943-949.

42. Dalgaard M, Thomsen C \& Hermansen K (2004) Effects of one single bout of low-intensity exercise on postprandial lipaemia in type 2 diabetic men. Br J Nutr 92, 469-476.

43. Gill JM, Al-Mamari A, Ferrell WR, et al. (2007) Effect of prior moderate exercise on postprandial metabolism in men with type 2 diabetes: heterogeneity of responses. Atherosclerosis 194, 134-143.

44. Tyldum GA, Schjerve IE, Tjønna AE, et al. (2009) Endothelial dysfunction induced by post-prandial lipemia: complete 
protection afforded by high-intensity aerobic interval exercise. J Am Coll Cardiol 53, 200-206.

45. Gill JMR (2004) Exercise and postprandial lipid metabolism an analysis of the current evidence. Eur J Lipid Sci Technol 106, 110-121

46. Magkos F, Wright DC, Patterson BW, et al. (2006) Lipid metabolism response to a single, prolonged bout of endurance exercise in healthy young men. Am J Physiol Endocrinol Metab 290, E355-E362.

47. Tsekouras YE, Yanni AE, Bougatsas D, et al. (2007) A single bout of brisk walking increases basal very low-density lipoprotein triacylglycerol clearance in young men. Metabolism 56, 1037-1043.

48. Zhang JQ, Thomas TR \& Ball SD (1998) Effect of exercise timing on postprandial lipemia and HDL cholesterol subfractions. J Appl Physiol 85, 1516-1522.

49. Gill JM, Frayn KN, Wootton SA, et al. (2001) Effects of prior moderate exercise on exogenous and endogenous lipid metabolism and plasma factor VII activity. Clin Sci (Lond) 100, 517-527.

50. Miyashita M, Burns SF \& Stensel DJ (2006) Exercise and postprandial lipemia: effect of continuous compared with intermittent activity patterns. Am J Clin Nutr 83, 24-29.

51. Cohen JC \& Berger GM (1990) Effects of glucose ingestion on postprandial lipemia and triglyceride clearance in humans. J Lipid Res 31, 597-602.

52. Westphal S, Leodolter A, Kahl S, et al. (2002) Addition of glucose to a fatty meal delays chylomicrons and suppresses VLDL in healthy subjects. Eur J Clin Invest 32, 322-327.

53. Whitley HA, Humphreys SM, Samra JS, et al. (1997) Metabolic responses to isoenergetic meals containing different proportions of carbohydrate and fat. Br J Nutr 78, 15-26.

54. Pedrini MT, Niederwanger A, Kranebitter M, et al. (2006) Postprandial lipaemia induces an acute decrease of insulin sensitivity in healthy men independently of plasma NEFA levels. Diabetologia 49, 1612-1618.

55. Chen YD, Skowronski R, Coulston AM, et al. (1992) Effect of acute variations in dietary fat and carbohydrate intake on retinyl ester content of intestinally derived lipoproteins. J Clin Endocrinol Metab 74, 28-32.

56. Cohen JC, Noakes TD \& Benade AJ (1988) Serum triglyceride responses to fatty meals: effect of fat content. Am J Clin Nutr 47, 825-827.

57. Dubois C, Armand M, Azais-Braesco V, et al. (1994) Effects of moderate amounts of emulsified dietary fat on postprandial lipemia and lipoproteins in normolipidemic adults. $\mathrm{Am} \mathrm{J}$ Clin Nutr 60, 374-382.

58. Dubois C, Beaumier G, Juhel C, et al. (1998) Effects of graded amounts $(0-50 \mathrm{~g})$ of dietary fat on postprandial lipemia and lipoproteins in normolipidemic adults. $A m J$ Clin Nutr 67, 31-38.

59. Murphy MC, Isherwood SG, Sethi S, et al. (1995) Postprandial lipid and hormone responses to meals of varying fat contents: modulatory role of lipoprotein lipase? Eur J Clin Nutr 49, 578-588.

60. Zammit VA (1996) Role of insulin in hepatic fatty acid partitioning: emerging concepts. Biochem J 314, 1-14.

61. Farese RV Jr, Yost TJ \& Eckel RH (1991) Tissue-specific regulation of lipoprotein lipase activity by insulin/glucose in normal-weight humans. Metabolism 40, 214-216.

62. Picard F, Naïmi N, Richard D, et al. (1999) Response of adipose tissue lipoprotein lipase to the cephalic phase of insulin secretion. Diabetes $\mathbf{4 8}, 452-459$.

63. Aarsland A, Chinkes D \& Wolfe RR (1996) Contributions of de novo synthesis of fatty acids to total VLDL-triglyceride secretion during prolonged hyperglycemia/hyperinsulinemia in normal man. J Clin Invest 98, 2008-2017.

64. Coppack SW, Jensen MD \& Miles JM (1994) In vivo regulation of lipolysis in humans. J Lipid Res 35, 177-193.

65. Frayn KN, Shadid S, Hamiani R, et al. (1994) Regulation of fatty acid movement in human adipose tissue in the postabsorptive-to-postprandial transition. Am J Physiol 266, 3 Pt 1, E308-E317.

66. Evans K, Burdge GC, Wootton SA, et al. (2002) Regulation of dietary fatty acid entrapment in subcutaneous adipose tissue and skeletal muscle. Diabetes 51, 2684-2690.

67. Karhunen LJ, Lappalainen RI, Tammela L, et al. (1997) Subjective and physiological cephalic phase responses to food in obese binge-eating women. Int J Eat Disord 21, 321-328.

68. LeBlanc J, Soucy J \& Nadeau A (1996) Early insulin and glucagon responses to different food items. Horm Metab Res 28, 276-279.

69. Goodner CJ, Walike BC, Koerker DJ, et al. (1977) Insulin, glucagon, and glucose exhibit synchronous, sustained oscillations in fasting monkeys. Science 195, 177-179.

70. Lang DA, Matthews DR, Peto J, et al. (1979) Cyclic oscillations of basal plasma glucose and insulin concentrations in human beings. $N$ Engl J Med 301, 1023-1027.

71. Hunter SJ, Atkinson AB, Ennis CN, et al. (1996) Association between insulin secretory pulse frequency and peripheral insulin action in NIDDM and normal subjects. Diabetes $\mathbf{4 5}$, 683-686.

72. Juhl C, Grøfte T, Butler PC, et al. (2002) Effects of fasting on physiologically pulsatile insulin release in healthy humans. Diabetes 51, Suppl. 1, S255-S257.

73. Gill JM, Herd SL, Tsetsonis NV, et al. (2002) Are the reductions in triacylglycerol and insulin levels after exercise related? Clin Sci (Lond) 102, 223-231. 